\title{
Effectiveness of Reiki Therapy on Management of Prehypertension among the Young Adults
}

\author{
Kanageswari Mangayarkarasi R .M.Sc (N) ${ }^{1}$, Pushpakala K.J.M.Sc $(\mathrm{N})^{2}$ \\ ${ }^{1}$ Tutor - Cardiothoracic Nursing department, Chettinad Hospital and Research Institute. \\ ${ }^{2}$ Associate professor- Cardio Thoracic Nursing department, Chettinad Hospital and Research \\ Institute, Tamilnadu. ,India.
}

\begin{abstract}
Cardio Vascular diseases remain as an important public challenge all over the world. Prevalence of these diseases has reached alarming proportions among Indians in recent years. The concept of pre hypertension was introduced as the new guideline for the management of blood pressure by Joint National Committee on prevention, detection, evaluation and treatment of high blood pressure. Complementary and Alternative therapies available today, which is acceptable with no side effects and remain as the supportive measure. One such is Reiki therapy. The aim of the study was to assess the effect of Reiki therapy in reducing the blood pressure among the young adults with Prehypertension.

A quantitative approach, True experimental, pre and post test design was used for this study. Using Simple random sampling technique 60 samples were selected and divided into two groups. The experimental group was provided with Reiki therapy over 30minutes per day for 15 days and no intervention for control group. Structured questionnaire and standard aneroid sphygmomanometer was used as tools for this study.

The findings revealed a significant change in blood pressure among the young adults with Prehypertension in experimental group who received Reiki therapy and minimal changes in control group with no intervention. The ' $t$ ' value of pre and post test $S B P$ and DBP, 11.04 and 7.72 was statistically significant at $p<0.05$ level, and in control group the ' $t$ ' value was 1.416 and 1.055 which was not significant at $p<0.05$ level. Thus the study proved that, Reiki therapy had effect on blood pressure which can be provided by the advance nurse practitioners as a complementary therapy in management of Prehypertension in daily practice.
\end{abstract}

Key words: Reiki therapy, Prehypertension, Blood pressure (SBP, DBP), Young adults.

\section{Introduction}

Health promotion is felt to be a cardinal principle of compassionate Nursing, being healthy physically and mentally brings a positive attitude among the people. Many people actively seek many ways to maintain and improve their health for a better life. The overall wealth of the world's population steadily increases and the people are aware that there is an inevitable trade-off with parallel increase in the total burden and impact of the disease of affluence. These diseases mark a transition in human history.

In developed countries, lower socio economic groups have a greater prevalence of risk factors, higher incidence of disease and higher mortality. World Health Organization (WHO) states that 17.3 million mortality rate from cardiovascular diseases in 2008; there will be about 20 million, Cardio vascular disease mortality by 2015 , accounting for $30 \%$ of all deaths worldwide. Hypertension is responsible for $57 \%$ of stroke and $24 \%$ of Coronary heart diseases in India.

About $15-37 \%$ of global adult population has Hypertension. Worldwide, high blood pressure is estimated to cause 7.1 million deaths; about $13 \%$ of total global fatality. The concept of pre hypertension was introduced as the new guideline for the management of blood pressure by Joint National Committee on prevention, detection, evaluation and treatment of high blood pressure. Pre hypertension is defined as systolic blood pressure of $120-139 \mathrm{mmHg}$ and diastolic pressure of $80-89 \mathrm{mmHg}$. .

The theme of World Health Day on April 7, 2013 was the need to control raised blood pressure (hypertension) as a "silent killer, global public health crisis". The slogan for the campaign was "Healthy Heart Beat, Healthy Blood Pressure". The WHO reports hypertension - which is both preventable and treatable contributes to the burden of heart diseases, stroke and renal failure, and is an important cause of premature death and disability. The organization estimates one in 3 adults has raised blood pressure.

The remarkable advances in therapy have provided ways to lower blood pressure in almost every person with hypertension. Nevertheless, hypertension continues to be a major public health problem whose prevalence is increasing worldwide, despite the therapeutic advances. People are turning to alternative, less invasive therapies in order to help them remedy health issues such as hypertension, heart diseases and chronic stress.

Complementary and alternative medicine (CAM) includes therapies such as acupuncture, dietary herbs, yoga, Meditation, Reiki, Music and laughter therapy helps in decreasing the blood pressure. Randomized 
clinical trials examine the clinical efficacy of Reiki healing touch as the supportive care modality for any medical condition. These therapies are used in reducing stress, anxiety, depression, pain and reducing the physiological parameters like heart rate, pulse rate and blood pressure.

A Preliminary study was conducted to assess the effect of Reiki healing on reducing the physiological parameters and came to conclusion that there was an actual physiological effect of the energy healing that would be triggered within the nervous system and more specifically the autonomic nervous system (ANS). The ANS is concerned with function such as respiration, blood pressure, sweating and vasodilatation. This study states that heart rate and diastolic blood pressure decreased significantly in people who received 30 minutes of Reiki therapy as compared to a placebo intervention or 30 minutes of rest given to the other groups.

Apart from this the investigator had the experience of practicing Reiki therapy to the patients in the hospitals with long term illness and general anxiety towards the invasive procedures and pharmacological treatment. Reiki therapy showed effectiveness on the patients experiencing such problems. The above literature and the personal experiences motivated the investigator to carry out the study and identify the effect of Reiki therapy on Prehypertension among the young adults.

\section{Objectives}

The objectives of the study were to

- assess the pretest level of blood pressure among young adults with prehypertension in experimental and control group in selected community area

- evaluate the effectiveness of Reiki therapy on young adults with prehypertension in experimental group in selected community area

- compare the pretest and post test level of blood pressure among young adults in both experimental and control group in selected community area

- associate the post test level of blood pressure with selected demographic variables in experimental group in selected community area

\section{Methods}

A Quantitative approach, True experimental pretest and post test design was selected for this study and conducted in Poonjeri a rural community located in outskirts of Chennai. A pilot study was conducted prior to the main study with 10 samples and feasibility and practicability of tools and methods was identified. The main study was conducted using 60 young adults by simple random sampling technique who was diagnosed with prehypertension. Among the young adults numbering was given and even numbers 30 samples were taken for experimental group and odd numbers for the control group. The objective of the study was explained and Informed consent was obtained from both the groups. Demographic data was collected by using structured questionnaire by visiting the samples residence. The experimental group was divided and Reiki therapy was given for 30minutes per day for 15 days. The level of blood pressure was assessed by means of standard aneroid sphygmomanometer five minutes before and five minutes after each Reiki therapy session and recorded.

Validity of the aneroid sphygmomanometer instrument was done by comparing the blood pressure measurements for four individuals with standard instrument used by the physician and found to be the same during each measurement. The tool was tested for reliability by using Test retest method.

\section{Data Collection And Analysis}

The study was approved by the Institutional Human Ethical Committee prior to the conduction of study. The aim of the study was explained to the study participants and informed consent was obtained by explaining in their own vernacular language. Descriptive statistics like mean, frequency, percentage were used for demographic data. Effect of Reiki therapy was evaluated by paired ' $t$ 'test and compared using independent' $t$ ' test and association of post test level of blood pressure with demographic variables was done using Chi square test. and' $\mathrm{P}$ 'value less than 0.05 was considered as statistically significant.

\section{Results And Discussion}

Participants in experimental group majority $47 \%$ of age $26-30$ years, $63 \%$ were males, $50 \%$ were educated in higher secondary level, $77 \%$ were married, $37 \%$ were self employed, $50 \%$ had habit of smoking, $33 \%$ had habit of alcohol and $57 \%$ with the history of hypertension. On the pre test assessment the mean systolic blood pressure was $130 \mathrm{mmof} \mathrm{Hg}$ ( SD 3.68) and diastolic blood pressure was $84 \mathrm{~mm}$ of $\mathrm{Hg}(\mathrm{SD} 2.29)$ in experimental group. In the control group the systolic and diastolic blood pressure was $132 / 86 \mathrm{~mm}$ of $\mathrm{Hg}$ with SD 3.68 and 2.49 respectively. The findings of the study revealed a significant change in Blood pressure among the young adults with Prehypertension in experimental group who received Reiki therapy and minimal changes in control group with no intervention. The' $t$ ' value 11.04 and 7.72 was statistically significant at $p<0.05$ level, and in control group the ' $t$ ' value was 1.416 and 1.055 which was not significant at $\mathrm{p}<0.05$ level. 
Table 1: Post test level of blood pressure among young adults after Reiki therapy in Experimental group

\begin{tabular}{|c|c|c|c|c|}
\hline TEST & $\begin{array}{c}\text { BLOOD } \\
\text { PRESSURE } \\
\text { ( } \mathrm{mm} \text { of } \mathrm{Hg} \text { ) }\end{array}$ & MEAN & $\begin{array}{l}\text { STANDARD } \\
\text { DEVIATION }\end{array}$ & 't' VALUE \\
\hline PRE TEST SBP & 130 & \multirow[t]{2}{*}{12.87} & \multirow[t]{2}{*}{6.38} & \multirow[t]{2}{*}{$11.04 *$} \\
\hline POST TEST SBP & 118 & & & \\
\hline PRE TEST DBP & 84 & \multirow[t]{2}{*}{6.93} & \multirow[t]{2}{*}{4.92} & \multirow[t]{2}{*}{$7.72 *$} \\
\hline POST TEST DBP & 78 & & & \\
\hline
\end{tabular}

* Significant at $\mathbf{p}<0.05$

Fig:1 Effectiveness of Reiki therapy in Management of Prehypertension among young adults in Experimental group.

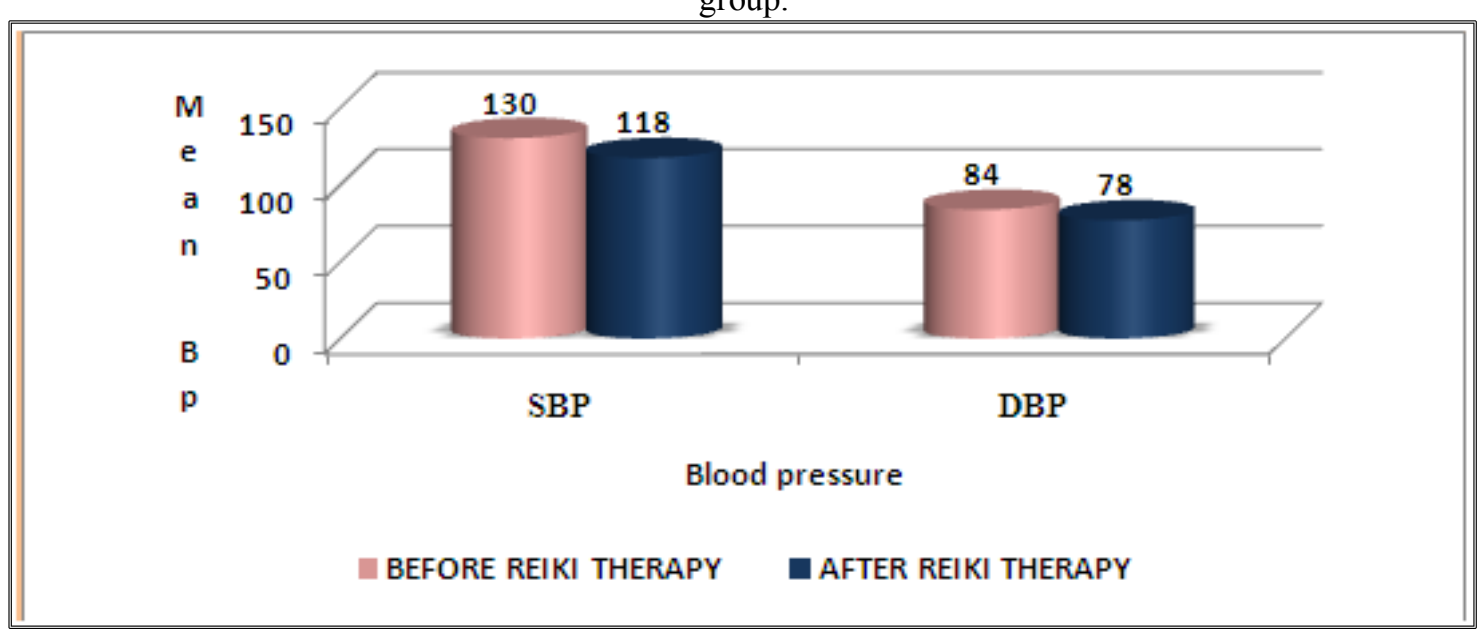

Results showed the difference in the blood pressure level among young adults with prehypertension after receiving Reiki therapy in experimental group. Data depicts that the mean post test level of blood pressure was lower than the pretest blood pressure level. The calculated' $t$ ' value for pre and post test systolic blood pressure is 11.04 and for pre and post test diastolic blood pressure is 7.72 which is greater than the table value. The systolic blood pressure during pretest was $130 \mathrm{~mm}$ of $\mathrm{Hg}$ and $118 \mathrm{~mm}$ of $\mathrm{Hg}$ during the post test. The diastolic blood pressure during pretest was $84 \mathrm{~mm}$ of $\mathrm{Hg}$ and $76 \mathrm{~mm}$ of $\mathrm{Hg}$ during the post test in experimental group. The computed ' $t$ ' value shows that there was a significant difference between the pre and posttest level of blood pressure. Based on these results the null hypothesis was rejected and the research hypothesis was accepted. This indicates that Reiki therapy was effective in management of prehypertension.

A similar study was conducted to find out the effectiveness of Reiki therapy in reducing the physiological parameters among the adults of age 20 to 50 years of age in hospital setting in United States. Forty-five (45) subjects assigned at random into three groups. Interventions: Three treatment conditions: no treatment (rest only); Reiki treatment by experienced Reiki practitioner, and placebo treatment by a person with no knowledge of Reiki and who mimicked the Reiki. Quantitative measures of autonomic nervous system function: such as heart rate, cardiac vagal tone, blood pressure, cardiac sensitivity to baroreflex, and breathing activity were recorded continuously for each heartbeat. Values during and after the treatment period were Compared with baseline data. Heart rate and diastolic blood pressure decreased significantly in the Reiki group Compared to both placebo and control groups. The study proved that Reiki therapy had some effect on the autonomic nervous System in reducing the physiological parameters. There was a significant difference between the pretest level of blood pressure and post test level of blood pressure in the experimental group which indicates that Reiki therapy can be provided as complementary therapy in management of prehypertension.

The study finding have shown that the post test level of blood pressure among young adults with prehypertension in experimental group with selected demographic variables had significant association for age, marital status, history of hypertension. A similar study was conducted to assess the prevalence of 
prehypertension and its risk factors among the north Indian population. The study included 716 young adults residing in the rural community area. The study concluded that young adults with family history of hypertension had increase in blood pressure and age had association with blood pressure.

\section{Implications For Nursing Practice And Research}

The present study will enable, nurses to apply theory into practice as the complementary and alternative therapies (CAM) are currently playing major part in clinical area and use this in their daily practice on patients to reduce the blood pressure. Nurses can conduct more research studies to demonstrate the effects of Reiki therapy along with other diseases. There is a need for extensive and intensive research in this area to provide evidence based care since the researches about this are in scarce.

\section{Conclusion}

In conclusion the discussion of the study findings shows that there was a significant difference in the level of blood pressure among young adults with prehypertension after receiving Reiki therapy. So it can be used as a complementary therapy in nursing practice.

\section{References}

[1]. Gupta R. (1997) Meta - analysis of prevalence of hypertension in India. Indian Heart Journal, 49:337-8

[2]. Goyal A,Yusuf F.(2006) The burden of cardiovascular disease in the Indian subcontinent. Ind J Med Res, 124:235-44.

[3]. World Health Organization. (2005) Preventing Chronic Diseases: A vital Investment. World Health Organization,Geneva,Switzerland: WHO global report.Retrived from http://www.who.int/chp/chronic disease report/en/

[4]. Whelan K.M.Wishnia GS.(2003).Reiki therapy:the benefits to a nurse/Reiki practitioner. Holistic Nurs Pract.17 (4):209-217.

[5]. Olson K, Hanson J. (1997) Using Reiki to manage pain: preliminary report, Cancer Prev Control.1 (2):108-113

[6]. Miles p, True G.(2003) Reiki- a review of biofield therapy history, theory, practice and research. Altern Ther Health Med. 9:62-71.

[7]. Lipinkski K.(2006) Finding Reiki:applications for your nursing practice. Beginnings.26 (1):6-7

[8]. American Holistic Nurses Association (2006).Position statements. Retrieved from http://www.ahna.org/home/home.html. 